\title{
ELECTROPRODUCTION OF STRANGENESS ON LIGHT NUCLEI
}

\author{
F. DOHRMANN ${ }^{c n}$, D. ABBOTT ${ }^{\text {b }}$, A. AHMIDOUCH ${ }^{\text {dfe }}$, P. AMBROZEWICZ ${ }^{\text {, }}$, \\ C.S. ARMSTRONG ${ }^{\text {bh }}$, J. ARRINGTON ${ }^{c i}$, R. ASATURYAN ${ }^{j}$, K. ASSAMAGAN ${ }^{f}$,

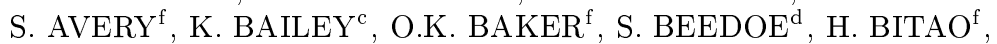 \\ H. BREUER ${ }^{\mathrm{k}}$, D.S. BROWN ${ }^{\mathrm{k}}$, R. CARLINI ${ }^{\mathrm{b}}, \mathrm{J}$ CHA $^{\mathrm{f}}, \mathrm{N} . \mathrm{CHANT}^{\mathrm{k}}$, \\ E. CHRISTY ${ }^{f}$, A. COCHRAN ${ }^{f}$, L. COLE ${ }^{f}$, G. COLLINS $^{k}$, C. COTHRAN ${ }^{1}$, \\ J. CROWDER ${ }^{\mathrm{m}}$, W.J. CUMMINGS ${ }^{\mathrm{c}}$, S. DANAGOULIAN ${ }^{\mathrm{db}}$, F. DUNCAN $^{\mathrm{k}}$,

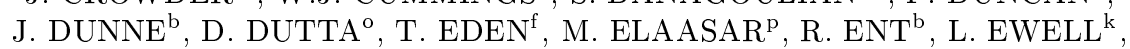 \\ H. FENKER ${ }^{\mathrm{b}}$, H.T. FORTUNE ${ }^{\mathrm{q}}$, Y. FUJII ${ }^{\mathrm{r}}$, L. GAN $^{\mathrm{f}}$, H. GAO $^{\mathrm{c}}$, K. GARROW $^{\mathrm{b}}$, \\ D.F. GEESAMAN ${ }^{c}$, P. GUEYE ${ }^{f}$, K. GUSTAFSSON ${ }^{k}$, K. HAFIDI ${ }^{c}$, \\ J.O. HANSEN ${ }^{c}$, W. HINTON ${ }^{f}$, H.E. JACKSON ${ }^{c}$, H. JUENGST ${ }^{s}$, C. KEPPEL ${ }^{f}$,

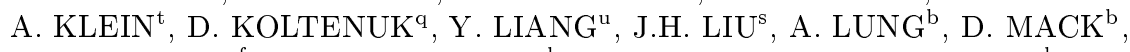 \\ R. MADEY ${ }^{f e}$, P. MARKOWITZ ${ }^{\text {ab }}$, C.J. MARTOFF ${ }^{\mathrm{g}}$, D. MEEKINS ${ }^{\mathrm{b}}$, \\ J. MITCHELL ${ }^{\mathrm{b}}$, T. MIYOSHI ${ }^{\mathrm{r}}, \mathrm{H}$. MKRTCHYAN ${ }^{\mathrm{j}}$, R. MOHRING $^{\mathrm{k}}$, \\ S.K. MTINGWA ${ }^{\mathrm{d}}$, B. MUELLER ${ }^{\mathrm{c}}$, T.G. O'NEILL ${ }^{\mathrm{c}}$, G. NICULESCU ${ }^{\mathrm{fv}}$, \\ I. NICULESCU ${ }^{\mathrm{f}}$, D. POTTERVELD ${ }^{\mathrm{c}}$, J.W. PRICE ${ }^{\mathrm{x}}$, B.A. RAUE ${ }^{\mathrm{ab}}$,

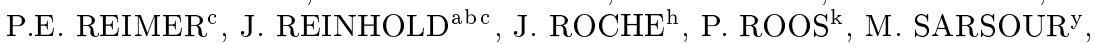 \\ Y. SATO ${ }^{r}$, G. SAVAGE ${ }^{f}$, R. SAWAFTA ${ }^{\mathrm{d}}$, R.E. SEGEL ${ }^{\circ}$, A.YU. SEMENOV $^{\mathrm{e}}$,

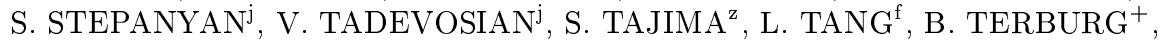

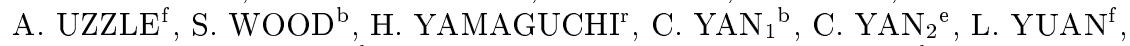 \\ M. ZEIER ${ }^{1}$, B. ZEIDMAN ${ }^{\mathrm{c}}$, AND B. ZIHLMANN ${ }^{1}$ \\ ${ }^{\mathrm{a}}$ Florida International University, ${ }^{\mathrm{b}}$ Thomas Jefferson National Accelerator \\ Laboratory, ${ }^{\mathrm{c}}$ Argonne National Laboratory, ${ }^{\mathrm{d}} \mathrm{NC}$ A $6 T$ State University, ${ }^{\mathrm{e}}$ Kent \\ State University, ${ }^{\mathrm{f}}$ Hampton University, ${ }^{\mathrm{g}}$ Temple University, ${ }^{\mathrm{h}}$ College of William \\ and Mary, ${ }^{\mathrm{i}}$ California Institute of Technology, ${ }^{\mathrm{j}}$ Yerevan Physics Institute, \\ ${ }^{\mathrm{k}}$ University of Maryland, ${ }^{\mathrm{l}}$ University of Virginia, ${ }^{\mathrm{m}}$ Juniata College, \\ ${ }^{\mathrm{n}}$ Forschungszentrum Rossendorf, ${ }^{\circ}$ Northwestern University, ${ }^{\mathrm{p}}$ Southern University \\ at New Orleans, ${ }^{\mathrm{q}}$ University of Pennsylvania, ${ }^{\mathrm{r}}$ Tohoku University, ${ }^{\mathrm{s}}$ University of \\ Minnesota, ${ }^{\mathrm{t}}$ Old Dominion University, ${ }^{\mathrm{u}}$ American University, ${ }^{\mathrm{v}}$ Ohio University, \\ ${ }^{\mathrm{w}}$ The George Washington University, ${ }^{\mathrm{x}}$ Rensselaer Polytechnic Institute, \\ ${ }^{\mathrm{y}}$ University of Houston, ${ }^{\mathrm{z}}$ Duke University, ${ }^{+}$University of Illinois
}

The $A\left(e, e^{\prime} K^{+}\right) Y X$ reaction has been investigated in Hall $\mathrm{C}$ at Jefferson Laboratory for 6 different targets. Data were taken for $Q^{2} \approx 0.35$ and $0.5 \mathrm{GeV}^{2}$ at a beam energy of $3.245 \mathrm{GeV}$ for ${ }^{1} \mathrm{H},{ }^{2} \mathrm{H},{ }^{3} \mathrm{He},{ }^{4} \mathrm{He}, \mathrm{C}$ and $\mathrm{Al}$ targets. The missing mass spectra are fitted with Monte Carlo simulations taking into account the production of $\Lambda$ and $\Sigma^{0}$ hyperon production off the proton, and $\Sigma^{-}$off the neutron. Models for quasifree production are compared to the data, excess yields close to threshold are attributed to FSI. Evidence for $\Lambda$-hypernuclear bound states is seen for ${ }^{3,4} \mathrm{He}$ targets. 


\section{Introduction}

The advent of high intensity $\mathrm{CW}$ electron beams at the Thomas Jefferson National Accelerator Facility provides the feasibility to study with high precision the electroproduction of strangeness as a complementary Ansatz to experiments with pion and kaon beams ${ }^{1}$. Jefferson Lab experiment E91016 measured the $A\left(e, e^{\prime} K^{+}\right) Y X$ for ${ }^{1} H,{ }^{2} H,{ }^{3} H e,{ }^{4} \mathrm{He}, \mathrm{C}$ and $\mathrm{Al}$ targets. Angular distributions of $K^{+}$were measured at forward angles with respect to the virtual photon, $\gamma^{*}$. Data for ${ }^{1} H$ and ${ }^{2} H$ targets have been presented previously $^{2,3,4}$, so that the data on Helium targets will be the focus of the present paper; the results are still preliminary.

\section{Experiment}

The experiment was performed in summer 1996 and fall 1999. The scattered electrons, $e^{\prime}$, were detected in the High Momentum Spectrometer (HMS) in coincidence with the electroproduced $K^{+}$, detected in the Short Orbit Spectrometer (SOS) in Hall C of Jefferson Lab. For a description of the experimental method see ${ }^{2}$. During the experiment the spectrometer angle for detecting the $e^{\prime}$ was kept fixed; the $K^{+}$arm was varied. For $A=1,2,3,4$ three different angle settings between the $\gamma^{*}$ and the ejected $K^{+}$were studied, $\theta_{\gamma^{*} K}^{l a b}=0^{\circ}$, $\simeq 6^{\circ}$, and $\simeq 12^{\circ}$. Since special high density cryogenic targets were used, the background, consisting of random coincidences as well as contributions from the aluminum walls of the targets cells were subtracted to obtain charge normalized yields.

\section{Results and Discussion}

The missing mass distribution for ${ }^{1} H\left(e, e^{\prime} K^{+}\right) Y$ shows two clearly resolved peaks corresponding to the $\Lambda$ and $\Sigma^{0}$ hyperons ${ }^{3,4}$. The two spectrometer coincidence acceptance as well as radiative processes are computed by Monte Carlo simulations. A parametrization of the $\gamma^{*} N$ cross section has been derived by fitting the kinematic dependences of the ${ }^{1} H\left(e, e^{\prime} K^{+}\right) Y$ cross section over the acceptance ${ }^{4}$; the same parametrization has been used for $A \geq 2$. For $A \geq 2$ we do not resolve separated $\Sigma^{0}, \Sigma^{-}$hyperon peaks, which are produced off the proton and the neutron, respectively. Moreover, for nuclear targets, the Fermi momentum and energy of the target nucleons have to be taken into account. Using the impulse approximation, we obtain momentum and in-medium energy of the struck nucleon in the nucleus from full spectral functions for the various targets, as provided by Benhar ${ }^{5}$. Excess yields close 

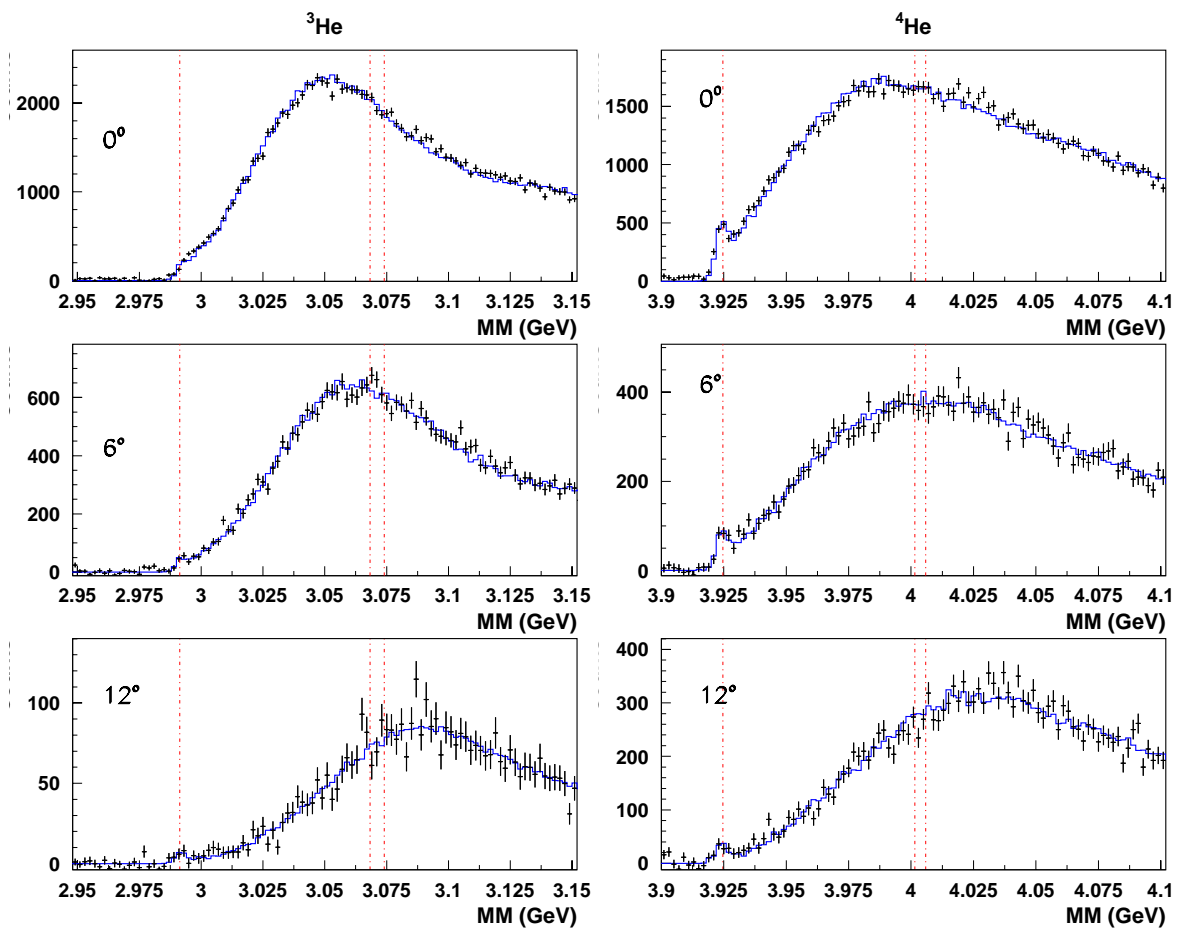

Figure 1. Missing mass distributions for ${ }^{3,4} \mathrm{He}\left(e, e^{\prime} K^{+}\right)$at $\theta_{\gamma^{*}, K}^{l a b}=0^{o}, 6^{o}, 12^{o}$. The solid line represents a Monte Carlo simulation of the qf contributions for $\Lambda, \Sigma^{0}, \Sigma^{-}$production off a nucleon in ${ }^{3,4} \mathrm{He}$. FSI corrections have been applied and the coherent production of ${ }^{3,4} H e\left(e, e^{\prime} K^{+}\right)_{\Lambda}^{3,4} H$ has been added as well. The dot-dashed vertical lines depict the threshold for quasifree $\Lambda, \Sigma^{0}$ and $\Sigma^{-}$production for $A=3$ and 4 .

to the respective $\Lambda n$ and $\Sigma N$ thresholds are attributed to FSI; for $A=2$ a more extensive study has been described in ${ }^{4}$. For $A=3,4$ we employ a simple effective range model of the FSI as described in ${ }^{6}$. For $A=3$ and 4 the agreement comparison between simulation and data is shown in Fig. 1. The separation of the two peaks for quasifree (qf) $\Lambda$ and $\Sigma$ production becomes less and less pronounced with increasing $A$. The foundation of the analysis for ${ }^{3,4} \mathrm{He}$ is described in ${ }^{7}$. In the regions of the qf $\Lambda$-thresholds for $A=3$ and 4, Fig. 1 exhibits relatively narrow enhancements at that we attribute to the ${ }_{\Lambda}^{3} H$ and ${ }_{\Lambda}^{4} H$ bound states. For both targets the structure is independent of the angle and is centered, within the resolution of the experiment, right at the correct binding energy. While barely discernible for ${ }^{3} \mathrm{He}$ at $\theta_{\gamma^{*}, K}^{l a b}=0^{\circ}$, the structure becomes more evident for $\theta_{\gamma^{*}, K}^{l a b}=6^{\circ}, 12^{\circ}$. It is clearly visible for 
all three measured angles for ${ }^{4} \mathrm{He}$. The resolution of the experiment does not allow for a separation of the ground and first excited states of ${ }_{\Lambda}^{4} H$, although the reaction mechanism favours the excited state. The preliminary analysis yields a cross section for the ${ }_{\Lambda}^{3} H$ state of a few $\mathrm{nb} / \mathrm{sr}$ and roughly $20 \mathrm{nb} / \mathrm{sr}$ for the ${ }_{\Lambda}^{4} H$ state. Further quantitative statements are expected after completing the analysis of the data.

\section{Summary}

The measurements on ${ }^{1} H\left(e, e^{\prime} K^{+}\right) Y$ established the basic high precision data to extend the experiments on associated hyperon production to nuclear targets. For $A \geq 2$ targets a full spectral function is used to describe the struck nucleon in the nucleus. In each case the kinematic model derived from hydrogen is used in impulse approximation to describe the qf production of hyperons off nuclear targets. Moreover, for $A=3,4$, we observe clear evidence for the ${ }_{\Lambda}^{3} H,{ }_{\Lambda}^{4} H$ bound states produced in electroproduction. After completing the analysis, we expect to obtain quantitative measurements of the electroproduction cross section for all of the targets studied: ${ }^{1} \mathrm{H},{ }^{2} \mathrm{H},{ }^{3} \mathrm{He},{ }^{4} \mathrm{He}, \mathrm{C}$, and Al.

\section{Acknowledgements}

This work was supported in part by the U.S. Department of Energy and the National Science Foundation. Support from Argonne National Laboratory and the U.S. Dept. of Energy under contract No. W-31-109-Eng-38 is gratefully ackowledged. The support of the staff of the Accelerator and Physics Division of Jefferson Lab is gratefully acknowledged. F. Dohrmann acknowledges the support by the the A.v.Humboldt Foundation through a Feodor Lynen Research Fellowship as well as the support by Argonne National Laboratory as the host institution for this research.

\section{References}

1. J.-M. Laget Nucl. Phys. A 691, 11c (2001)

2. D. Abbott et al, Nucl. Phys. A 639, 197c (1998).

3. B. Zeidman et al, Nucl. Phys. A 691, 37c (2001).

4. J. Cha, PhD thesis, Hampton University, 2000.

5. O. Benhar et al, Nucl. Phys. A 579, 493 (1994).

6. D. Gaskell et al, Phys. Rev. Lett. 87, 202301 (2001).

7. A. Uzzle, PhD thesis, Hampton University 2002. 